\title{
Prediction method for nutritional quality of Korla pear during storage
}

\author{
Yang Liu' ${ }^{1,2}$, Qiang Zhang ${ }^{3}$, Hao Niu², Hong Zhang², Haipeng Lan², Yong Zeng ${ }^{2}$, Fuguo Jia ${ }^{1 *}$ \\ (1. College of Engineering, Northeast Agricultural University, Harbin 150030, China; \\ 2. College of Mechanical and Electrical Engineering, Tarim University, Alar, Xinjiang 843300, China; \\ 3. College of Engineering, Huazhong Agricultural University, Wuhan 430070, China)
}

\begin{abstract}
It is difficult to control the quality of Korla pear with different degrees of maturity during storage. Here, a method was proposed for predicting the effects of harvest maturity and cold storage time on the quality indices (soluble solid content (SSC) and Vitamin $\mathrm{C}(\mathrm{Vc})$ content) of Korla pear. The generalized regression neural network (GRNN) and adaptive neuro-fuzzy inference system (ANFIS) were employed to predict the quality changes of Korla fragrant pear fruit during storage. The results demonstrated that during cold storage the SSC in pears with 10\%-70\% harvest maturity showed continuous increases in the first $90 \mathrm{~d}$ of storage and then a slight decline thereafter, while that in pears with $80 \%$ and $90 \%$ harvest maturity exhibited slow decreases throughout the storage process. With the extension of storage time, the Vc content of pears with $10 \%-90 \%$ harvest maturity showed continuous decreases. The harvest maturity of Korla pear was extremely positively correlated with SSC and Vc content $(p<0.01)$ in a given storage period. Storage time showed an extremely significant negative correlation with the $\mathrm{Vc}$ content $(p<0.01)$ at the $40 \%-90 \%$ harvest maturity and an significant negative correlation with the $\mathrm{Vc}$ content $(p<0.05)$ at the $10 \%-30 \%$ harvest maturity. At the $10 \%-70 \%$ harvest maturity, storage time showed a significant positive correlation with the SSC $(p<0.05)$. The trained model could well predict the variation trend of quality indices of pear fruit during storage. The ANFIS with the input membership function of gbellmf had the best performance in predicting the SSC $\left(R M S E=0.175 ; R^{2}=0.98\right)$, and that with the input membership function of trimf exhibited the best performance in predicting Vc content $\left(R M S E=0.075 ; R^{2}=0.99\right)$. The research findings can provide reference for predicting the fruit nutritional quality at delivery and decision-making on the storage time of Korla fragrant pear.
\end{abstract}

Keywords: Korla fragrant pear, harvest maturity, storage time, nutritional quality, prediction method DOI: $10.25165 /$ j.ijabe.20211403.5990

Citation: Liu Y, Zhang Q, Niu H, Zhang H, Lan H P, Zeng Y, et al. Prediction method for nutritional quality of Korla pear during storage. Int J Agric \& Biol Eng, 2021; 14(3): 247-254.

\section{Introduction}

Korla pear is an agricultural product of national geographical indication $^{[1,2]}$. In general, it is harvested centrally and stored chronically. How to guarantee the storage quality of Korla pear is still critically important but very challenging. Storage in refrigeration houses under cold storage temperature after harvest and then marketing at proper time have been the main way to prolong the supply and promote the economic value of Korla pear ${ }^{[3]}$. At present, the delivery time of Korla pear from warehouse is mainly determined by market supply-demand conditions and subjective experiences. However, it is far from accurate to determine the quality changes of pears during storage based on subjective experiences, which possibly results in poor quality at delivery and consequently loss of economic values of the products. In particular, the nutritional quality of Korla pear cannot be

Received date: 2020-06-29 Accepted date: 2020-11-11

Biographies: Yang Liu, Master, Lecturer, research interests: agricultural products processing technology, Email: hxtxylove@126.com; Qiang Zhang, $\mathrm{PhD}$, Lecturer, research interests: agricultural products processing technology, Email: zq604@mail.hzau.edu.cn; Hao Niu, Master, Lecturer, research interests: agricultural products processing technology, Email: 18167528125@163.com; Hong Zhang, Master, Professor, research interests: agricultural machinery and equipment design, Email: zhghog@163.com; Haipeng Lan, PhD, Associate Professor, research interests: agricultural products processing technology, Email: lanhaipeng@126.com; Yong Zeng, PhD, Associate Professor, research interests: agricultural products processing technology, Email: 15504577650@126.com.

*Corresponding author: Fuguo Jia, PhD, Professor, research interest: agricultural products processing technology. College of Engineering, Northeast Agricultural University, 600 Changjiang Road, Harbin 150030, China. Tel: + 86 451-55190620, Email: jiafg301@neau.edu.cn. determined during storage, which results in the decrease of the commodity value of Korla pear with high nutrition content. Therefore, clarifying the variation laws of the nutritional quality of Korla pear during storage and establishing a reliable nutritional quality prediction model can provide important guidance for the control of delivery quality and delivery time, which is of great significance in real production of Korla pear.

Korla pear is a kind of food, so its nutritional value should be first considered. Nutritional value of pears is characterized by soluble solid content (SSC) and Vitamin C (Vc) content which are often used as the main nutritional quality indices ${ }^{[4,5]}$. In order to control nutritional quality of pears during storage, many studies have been carried out on the variation laws of SSC and Vc content of pears. For example, Wang et al. ${ }^{[6]}$ demonstrated that storage temperature could significantly influence SSC and Vc content of Korla pear. Rizzolo et al. ${ }^{[7]}$ indicated that SSC was higher in controlled atmosphere and dynamic controlled atmosphere than in air during storage. Jia et al. ${ }^{[8]}$ demonstrated that 1-methylcyclopropene (1-MCP) could significantly inhibit the decline of Vc content but had no significant effect on SSC. These studies were mainly focused on the influence of storage conditions on fruit quality, but the effect of harvest maturity, which is also critical to the quality of fruit during storage, has been largely ignored $^{[9]}$. Therefore, some scholars have discussed the influence of harvest maturity on the quality of postharvest pear fruits. For instance, Boonyakiat et al. ${ }^{[10]}$ found that harvest maturity has great impacts on the rotting rate and quality changes of d'Anjou pear. Blanckenberg et al. ${ }^{[11]}$ analyzed the behaviors of consumers and found that maturity affected the quality of Forelle pear, Packhams Triumph pear and Abate Fetel pear, thus influencing the 
consumers' purchase selection. It is important to note that pear fruits suffer from quality decline along with storage time. Dong et al. ${ }^{[12]}$ demonstrated that treatment with 1 -MCP still cannot maintain the quality of the pears after long-term storage. Therefore, the storage time limit, which is largely determined by the maturity of pear fruits, should be fully considered under all storage modes ${ }^{[13]}$. Harvest maturity and storage time are two important factors that influence the storage quality of pears. It is extremely important to reveal the variation laws of pear quality at different harvest maturity and storage time and develop quality prediction models.

Recently, generalized regression neural network (GRNN) and adaptive neuro-fuzzy inference system (ANFIS) have been widely applied to study the quality of fruits and vegetables owing to their high training speed and prediction accuracy ${ }^{[14]}$. For example, they have been applied to the prediction of SSC in apples and ascorbic content in fresh pineapple, and used to construct a damage detection and classification model of Myrica rubra ${ }^{[15-17]}$. Nevertheless, there has been little research on their application in predicting the nutritional quality of pears under storage.

In sum, the objectives of this research are (1) to study the the variation law of SSC and Vc content in Korla pear at different levels of harvest maturity during cold storage, (2) to construct a GRNN model and an ANFIS model for predicting SSC and Vc content, and (3) to compare the performance of the two models in predicting SSC and Vc content to determine the best prediction model.

\section{Materials and method}

\subsection{Sample preparation}

With reference to the national and industrial standards ${ }^{[18,19]}$, Korla pear samples were collected from the Pear Garden of the Alar City $12^{\text {th }}$ Regime, the first Division of Xinjiang Production Construction Group from August 31st to October 2nd, 2018. Pear fruits with perfect surface, no diseases and insects, and uniform size were collected (100 samples every day). Then, 20 fruits were randomly chosen for the measurement of quality indices. The mean index values were recorded and the harvest maturity was calculated according to the quantitative evaluation equation of pear maturity ${ }^{[20]}$. Samples with the same maturity were transferred to the refrigeration house and stored under cold storage temperature (temperature $=(-1 \pm 1)^{\circ} \mathrm{C}$; relative humidity $=90 \% \pm 5 \%$ ). During the storage, all samples were marked and classified according to nine maturity levels from $10 \%-90 \%$.

\subsection{Measurement of quality indices}

During storage, the quality indices of pear samples at nine maturity levels were tested every $30 \mathrm{~d}$. For each maturity level, 10 pear samples were randomly chosen for the measurement of SSC and Vc content, respectively, and the average value was taken for each index.

\subsubsection{SSC}

SSC is one of the most important internal properties because it is a key parameter for determining the nutritional quality and flavour $^{[21,22]}$, and it also could provide valuable information for commercial decision making ${ }^{[23,24]}$. Zero-point alignment of the PAL-1 digital refractometer (ATAGO Company, Japan) was performed with distilled water. About $1 \mathrm{~cm}$ thick pulp was cut below the fruit skin every $120^{\circ}$ along the equator of fruit and then put in 3-layer gauze to squeeze the juice artificially. Measuring points for SSC of the pear fruit is shown in Figure 1. The juice was filtered and then dropped into the test window of the refractometer. Three SSC values (\%) were recorded and the mean was calculated as the final SSC value of the samples.

\subsubsection{Vc content}

Vc can represent the nutritional value of the fruit, have an antioxidant effect ${ }^{[25]}$, delay the aging process of fruit after harvest ${ }^{[25]}$, and have been associated with the prevention of chronic diseases in humans ${ }^{[5]}$. The samples were collected in three points in an equal distance along the equator of the fruit. Measuring points for $\mathrm{Vc}$ content of the pear fruit is shown in Figure 1. Next, $100 \mathrm{~g}$ pulp was collected by an electronic analytical balance into a mortar and ground into slurry with $2 \%$ oxalic acid. Then, $30 \mathrm{~g}$ of slurry was collected for the later test by 808 Titrando automatic potentiometric titrator (Vantone Company, Switzerland). The slurry was titrated by using 2,6-dichlorophenolindophenol solution, and test was ended at titration endpoint. Three $\mathrm{Vc}$ content values were recorded and the mean was calculated as the final $\mathrm{Vc}$ content value of the samples. The results were expressed in $\mathrm{mg} / 100 \mathrm{~g}$.

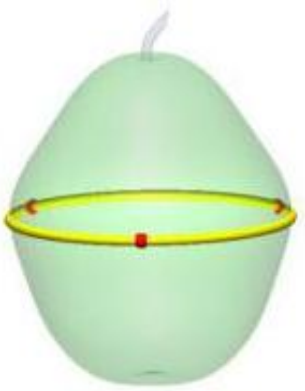

Figure 1 Measuring points (denoted by $\mathbf{m}$ ) for quality indices of the pear fruit

\subsection{Determination of harvest maturity}

The maturity of samples was determined by the quantitative evaluation equation of Korla pear proposed by Lan et al. ${ }^{[20]}$. However, pears with the same growth laws might have different qualities due to different meteorological conditions and field managements in the producing areas ${ }^{[26]}$. Therefore, it is necessary to calibrate the evaluation equation first before the evaluation of the fruit maturity. By analyzing the maturation laws of pears, this method determines the variation trend of quality indices during the maturation of pears using the following equation:

$$
M_{i}=\left|\frac{y_{i}-y_{1}}{y_{2}-y_{1}}\right| \times 100 \%
$$

where, $M_{i}$ is the maturity, $\% ; y_{1}$ is the initial value of the indices; $y_{2}$ is the final value of the indices and $y_{i}$ is the value of a particular index.

In this experiment, the variation ranges of SSC and $\mathrm{Vc}$ content were $11.48 \%-15.36 \%$ and $1.83-6.05 \mathrm{mg} / 100 \mathrm{~g}$, respectively. The maturity evaluation equation was calibrated according to Equation (2) and Equation (3). No matter we use Equation (2) or Equation (3) , the results are identical for determining maturity.

$$
\begin{aligned}
& M_{S S C}=3.958-0.258 y_{S S C} \\
& M_{V c}=0.237 y_{V c}-0.434
\end{aligned}
$$

where, $M_{s s c}$ is the harvest maturity determined by SSC, \%; $y_{S S C}$ is the SSC value during harvest period, $\% ; M_{V c}$ is the harvest maturity determined by $\mathrm{Vc}$ content, $\% ; y_{V c}$ is the $\mathrm{Vc}$ content value during harvest period, $\mathrm{mg} / 100 \mathrm{~g}$.

\subsection{Modeling method}

Two modeling methods, GRNN and ANFIS, were applied in the present study. On this basis, the quality prediction models of Korla pear with different harvest maturity during storage were 
constructed.

\subsubsection{GRNN model}

GRNN is an improved model of radial basis function neural network, and is a feed forward neural network model based on nonlinear regression theory. It is characterized by good simulation effects, fast calculation and stable results, and shows great advantages over the training set of small sample size ${ }^{[27]}$. GRNN is composed of an input layer, a pattern layer, a summing layer and an output layer. The structure of GRNN is shown in Figure 2.

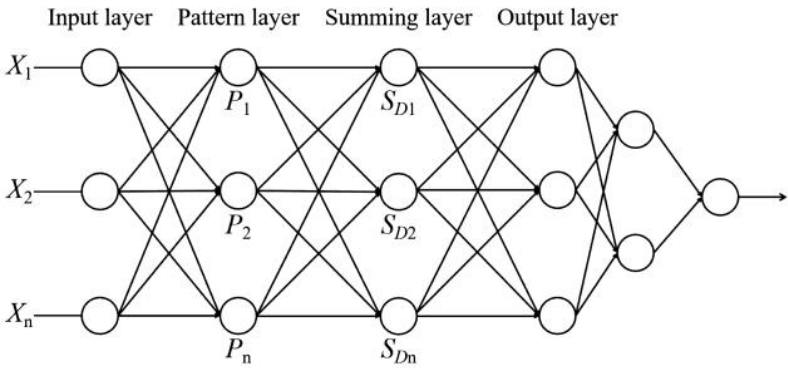

Figure 2 Basic structure of GRNN model

In the GRNN model, the harvest maturity of pears and storage time were used as the network input, and the quality indices of pears were set as the network output. According to the statistics of experimental data, a total of 45 groups of datasets were gained, from which $70 \%$ experimental data were randomly chosen for training set and the rest 30\% were chosen for testing set. This is also the common practice of most researchers to ensure the amount of modeling data and prediction data and make the model robust ${ }^{[28,29]}$. Next, the most important parameter smooth factor $\sigma$ of the GRNN model was determined by a cross-validation search algorithm. The value of $\sigma$ determines whether the radial basis neurons can respond to the interval of input variables.

\subsubsection{ANFIS mode}

The essence of ANFIS is to use the relatively mature parameter learning algorithm in neural network, namely the back propagation algorithm or a back propagation algorithm of hybrid least square estimation $^{[30]}$. The shape parameters of membership functions (MFs) of variables in ANFIS are adjusted by learning a given group of input-output dataset. The structure of the ANFIS model is shown in Figure 3.

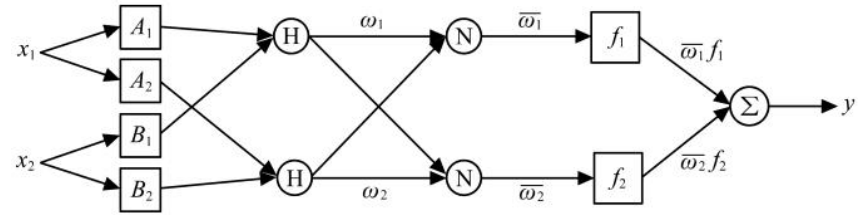

Figure 3 General ANFIS architecture

In order to ensure the amount of modeling data and prediction data and make the model robust, $70 \%$ and $30 \%$ experimental data were randomly assigned to the training set and testing set of ANFIS model, respectively. The ANFIS tool kit of Matlab 2017 was applied for the modeling. According to the experimental results, the input and output parameters were chosen: 1) system inputs: harvest maturity and storage time; 2) system output: quality indices. The training set was first input, and then an initial fuzzy inference system was generated by the selection grid method, as is shown in Figure 4.

A total of eight types of input MFs were applied, including trimf, trapmf, gbellmf, gaussmf, gasuss $2 \mathrm{mf}$, pimf, dsigmf and psigmf. Linear MFs were chosen for the output. The parameter settings of the input and output of the MFs determine the prediction results of the model, and thus have great significance in the prediction of quality changes of fruits.

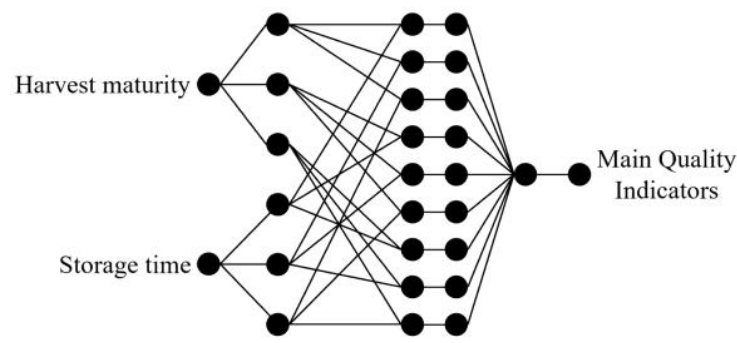

Figure 4 Developed ANFIS structure

2.4.3 Determination of the optimal prediction model

To obtain the best prediction model, the prediction performance of the constructed models was assessed by the root-mean-square error (RMSE) and coefficient of linear regression line $\left(R^{2}\right)$. The $R M S E$ was calculated as follows:

$$
R M S E=\sqrt{\sum_{i}^{N} \frac{\left(K_{E}-K_{P}\right)^{2}}{N}}
$$

where, $N$ is the total number of data; $K_{P}$ is the predicted value of the model output, and $K_{E}$ is the observed value.

A good model should be equipped with a lower RMSE value. Besides, the model has good performance if the value of $R^{2}$ is within the range of $0.82-0.90$, whereas a $R^{2}$ value higher than 0.90 indicates that the model is sufficient to meet specific goals of prediction.

\section{Experimental results and analysis}

\subsection{Variation law of pear quality in the storage process}

3.1.1 Variation law of SSC in the storage process

Figure 5 shows the variation law of SSC in Korla pear at different levels of harvest maturity during storage. Generally, SSC increased firstly and then decreased during the storage. The pears with higher harvest maturity had higher SSC than those with lower harvest maturity throughout the storage period. Specifically, the SSC in pears with $10 \%-70 \%$ harvest maturity showed continuous increases in the first $90 \mathrm{~d}$ of storage and then a slight decline thereafter, while that in pears with $80 \%$ and $90 \%$ harvest maturity exhibited slow decreases throughout the storage process.

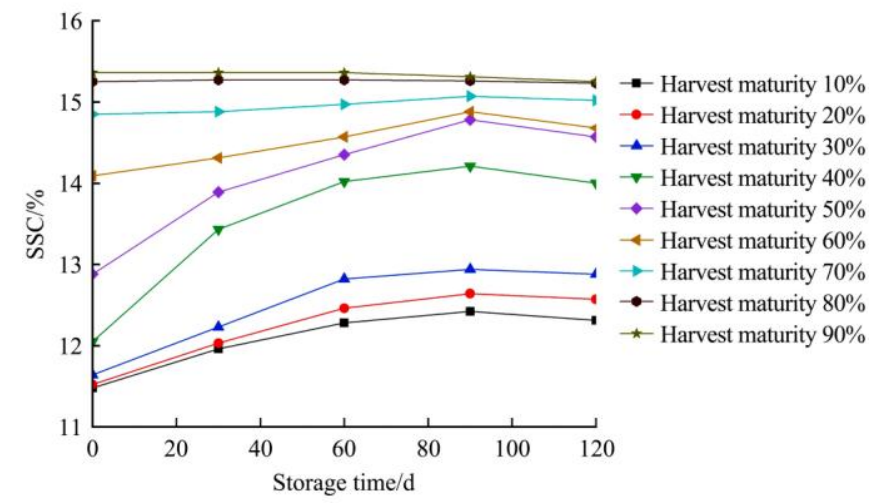

Figure 5 Relationship between SSC and storage time

In the growth stage of pears, SSC increases continuously with the development of fruit, particular at the middle and late growth stages, and reaches the maximum when the fruit is completely mature $^{[31]}$. Therefore, high-maturity pears have higher SSC than low-maturity pears. Since pear is a typical kind of climacteric fruits, the starch in the fruit can be transformed to soluble sugar in the early period of storage to satisfy the energy demand by 
respiration, which can explain the continuous increases in SSC at the early storage stage. However, the soluble sugar transformed from starch cannot provide enough energy for the gradually enhanced respiration in the late storage period, resulting in continuous decline of $\mathrm{SSC}^{[32]}$.

\subsubsection{Variation laws of $\mathrm{Vc}$ content in the storage process}

Figure 6 shows the variation laws of Vc content in Korla pear at different harvest maturity with storage time. Clearly, there were great differences between high-maturity and low-maturity pears in term of $\mathrm{Vc}$ content in the early storage process. High-maturity pears had higher Vc content than low-maturity pears throughout the storage process. Besides, with the extension of storage time, the $\mathrm{Vc}$ content of pears with different levels of maturity showed continuous decreases.

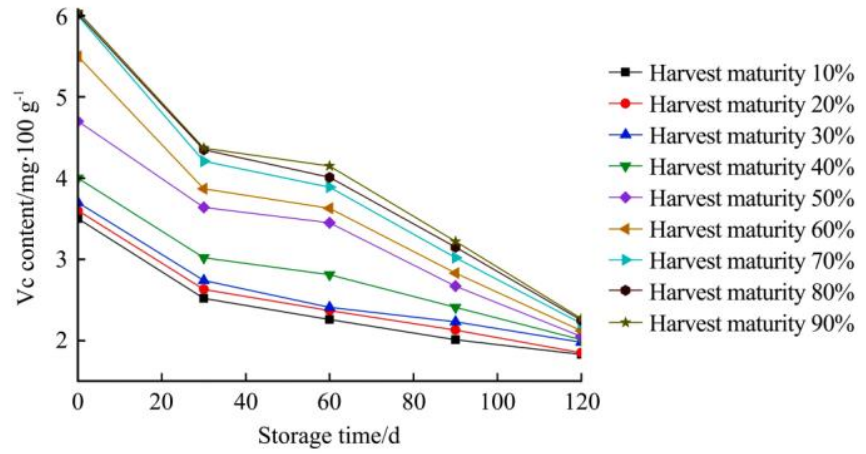

Figure 6 Relationship between Vc content and storage time

$\mathrm{Vc}$ accumulates quickly in pears at the late growth stage. However, low-maturity pear fruits were harvested before the complete synthesis of $\mathrm{Vc}$ and lost nutrient supply from the tree, resulting in their lower $\mathrm{Vc}$ content than high-maturity pear fruits ${ }^{[33]}$. Pears suffer from great $\mathrm{Vc}$ loss after long-time storage. Low-maturity pears can maintain $\mathrm{Vc}$ content, while high-maturity pears consume Vc quickly ${ }^{[34]}$. Therefore, high-maturity pears showed a more dramatic decrease in Vc content than low-maturity pears.

3.2 Correlations of harvest maturity and storage time with quality indices

Correlation analysis results of storage time and harvest maturity with quality indices are listed in Table 1 and Table 2. According to the general analysis, the harvest maturity of Korla pear was extremely positively correlated with SSC and Vc content $(p<0.01)$ in a given storage period. At the $40 \%-90 \%$ harvest maturity, storage time showed an extremely significant negative correlation with the $\mathrm{Vc}$ content $(p<0.01)$. At the 10\%-30\% harvest maturity, storage time showed an significant negative correlation with the Vc content $(p<0.05)$. And at the $10 \%-70 \%$ harvest maturity, storage time showed a significant positive correlation with the SSC $(p<0.05)$. To sum up, there are certain internal relations among harvest maturity, storage time and quality indices of pears.

Table 1 Correlations between harvest maturity and quality indices of Korla pear within the same storage time

\begin{tabular}{ccc}
\hline $\begin{array}{c}\text { Storage time } \\
\text { /d }\end{array}$ & $\begin{array}{c}\text { Correlation between harvest } \\
\text { maturity and SSC }\end{array}$ & $\begin{array}{c}\text { Correlation between harvest } \\
\text { maturity and Vc content }\end{array}$ \\
\hline 0 & $0.943^{* *}$ & $0.929^{* *}$ \\
30 & $0.980^{* *}$ & $0.976^{* *}$ \\
60 & $0.974^{* *}$ & $0.979^{* *}$ \\
90 & $0.941^{* *}$ & $0.981^{* *}$ \\
120 & $0.957^{* *}$ & $0.973^{* *}$ \\
\hline
\end{tabular}

Note: ** represents extremely significant correlation $(p<0.01)$
Table 2 Correlations between storage time and quality indices of Korla pear at the same harvest maturity

\begin{tabular}{ccc}
\hline Maturity/\% & $\begin{array}{c}\text { Correlation between storage } \\
\text { time and SSC }\end{array}$ & $\begin{array}{c}\text { Correlation between storage } \\
\text { time and Vc content }\end{array}$ \\
\hline 10 & $0.874^{*}$ & $-0.931^{*}$ \\
20 & $0.917^{*}$ & $-0.947^{*}$ \\
30 & $0.906^{*}$ & $-0.942^{*}$ \\
40 & $0.879^{*}$ & $-0.971^{* *}$ \\
50 & $0.891^{*}$ & $-0.939^{* *}$ \\
60 & $0.894^{*}$ & $-0.968^{* *}$ \\
70 & $0.912^{*}$ & $-0.979^{* *}$ \\
80 & -0.469 & $-0.983^{* *}$ \\
90 & -0.734 & $-0.964^{* *}$ \\
\hline
\end{tabular}

Note: * refers to significant correlation $(p<0.05)$ and $* *$ represents extremely significant correlation $(p<0.01)$.

\subsection{Prediction model of pear quality indices}

3.3.1 Prediction of SSC and Vc content in Korla pear based on GRNN model

According to the analysis of the training results of GRNN model, the optimal smooth factor $\sigma$ for predicting the SSC of pears was 0.36 and that for predicting the Vc content was 0.34 , when the model showed the best prediction performance and the smallest error. Subsequently, the remaining data were input into the two trained models, resulting in two groups of prediction values. Through linear fitting between the observed and predicted values, it was found that the $R^{2}$ and RMSE of the GRNN model were 0.97 and 0.28 in predicting the SSC of pears, and 0.97 and 0.30 in predicting the Vc content, respectively. The fitting results are shown in Figure 7. These results demonstrate that the trained GRNN model can effectively predict the relations of SSC and Vc content with the harvest maturity and storage time of pears.

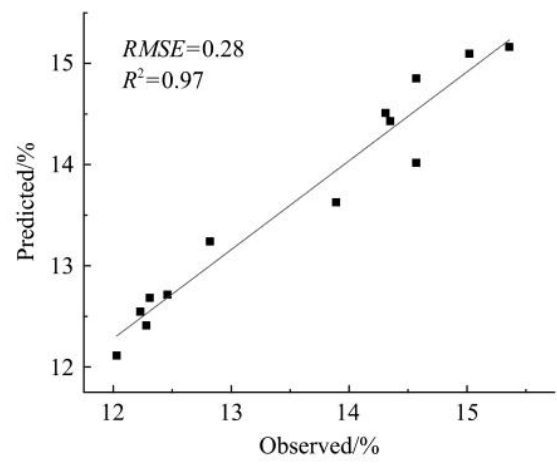

a. SSC

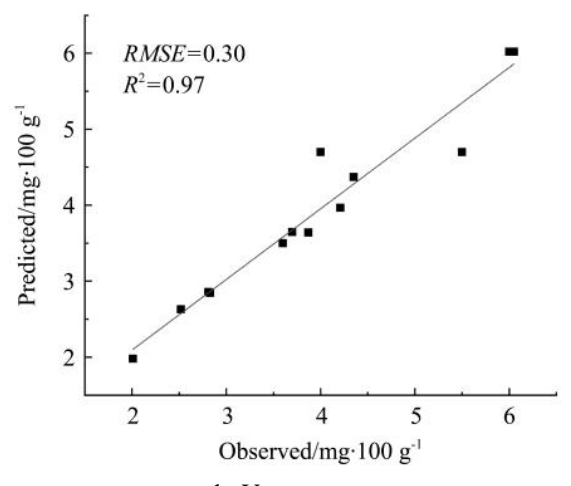

b. Vc content

Figure 7 Relationships between observed and predicted values of quality indices of Korla pear by the GRNN models

3.3.2 Prediction of SSC in Korla pear based on ANFIS model

In the model training stage, the initial ANFIS model was generated by the meshing method and eight types of MFs were 
applied for the fuzzification of the input data. Hybrid learning can help to perfect the parameters of ANFIS model in each stage to decrease the errors to the maximum extent.

After the training stage, the ANFIS model was tested on an independent dataset. The correlations between the observed values and predicted values obtained from model training and prediction are shown in Figure 8. In addition, the RMSE and $R^{2}$ values of the training set and testing set are listed in Table 3 .
Under all MFs, the $R^{2}$ values between the observed and predicted values are higher than 0.96 , implying that the trained ANFIS model can predict the variation trend of SSC in Korla pear during storage. In addition, further analysis showed that the ANFIS model with gbellmf as the input MF had better performance than that with other MFs. Hence, the ANFIS model based on gbellmf can be used to well predict the changes of SSC in pears throughout the storage process.

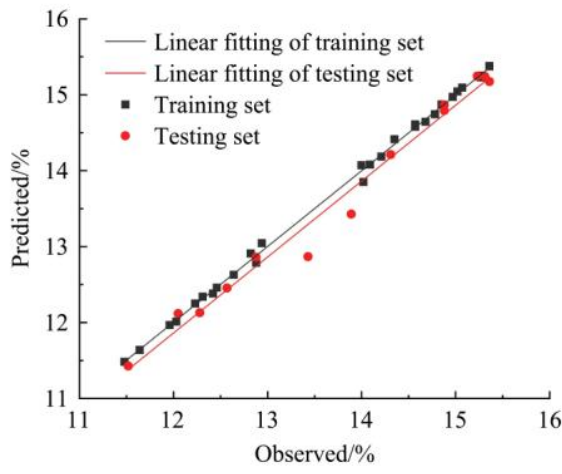

a. trimf

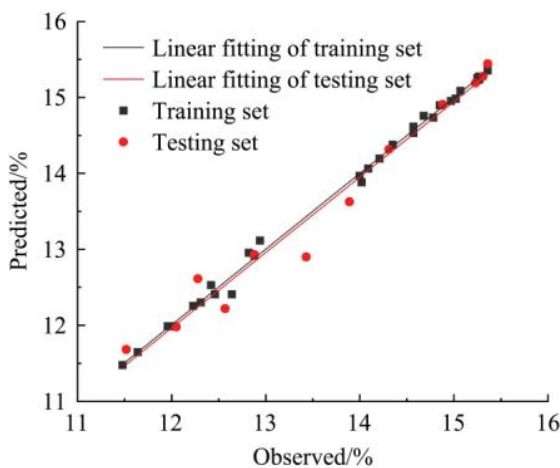

d. gaussmf

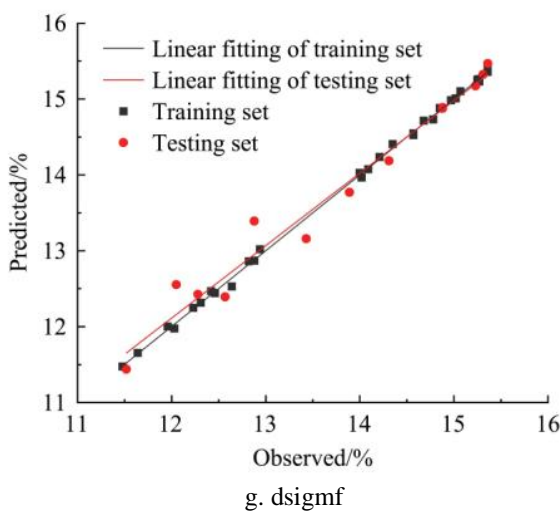

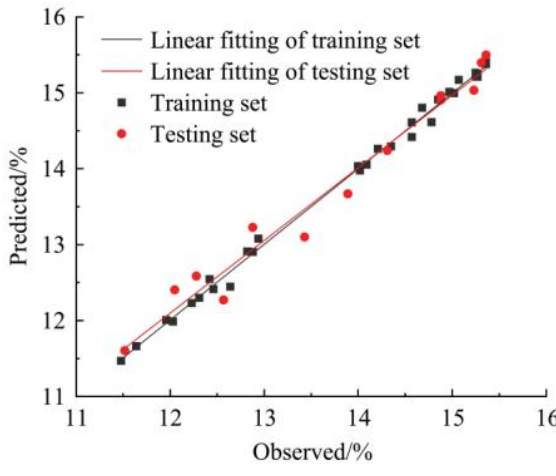

b. trapmf

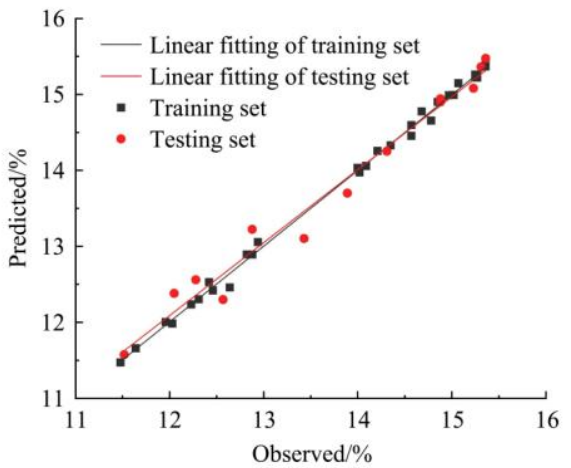

e. gasuss $2 \mathrm{mf}$

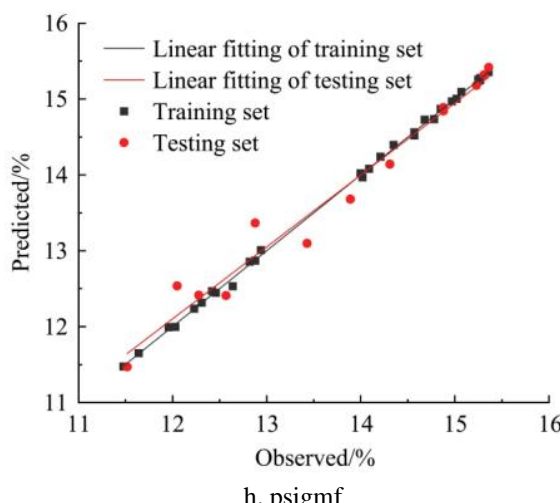

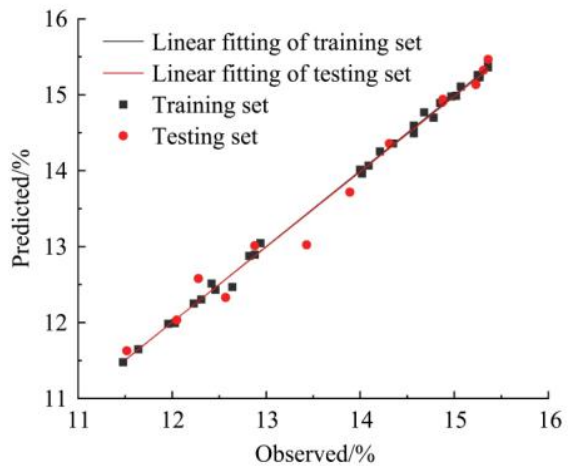

c. gbellmf

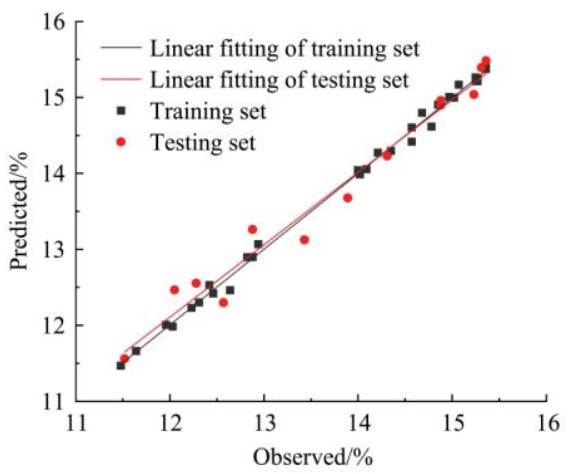

f. $\operatorname{pimf}$

Figure 8 Correlations between observed and predicted SSC of Korla pear during storage by the ANFIS model with different MFs at training and prediction phases

Table 3 Root mean squared error (RMSE) and coefficient of determination $\left(R^{2}\right)$ for the prediction of SSC by the ANFIS model with different input MFs at the training and prediction phases

\begin{tabular}{|c|c|c|c|c|}
\hline \multirow{2}{*}{$\begin{array}{l}\text { Membership } \\
\text { functions }\end{array}$} & \multicolumn{2}{|c|}{ Training stage } & \multicolumn{2}{|c|}{ Prediction stage } \\
\hline & RMSE & $R^{2}$ & $R M S E$ & $R^{2}$ \\
\hline trimf & 0.049 & 0.99 & 0.222 & 0.98 \\
\hline trapmf & 0.078 & 0.99 & 0.227 & 0.97 \\
\hline gbellmf & 0.054 & 0.99 & 0.175 & 0.98 \\
\hline gaussmf & 0.071 & 0.99 & 0.220 & 0.97 \\
\hline gasuss $2 \mathrm{mf}$ & 0.064 & 0.99 & 0.210 & 0.97 \\
\hline pimf & 0.074 & 0.99 & 0.228 & 0.97 \\
\hline dsigmf & 0.039 & 0.99 & 0.231 & 0.97 \\
\hline psigmf & 0.034 & 0.99 & 0.233 & 0.96 \\
\hline
\end{tabular}




\subsubsection{Prediction of $\mathrm{Vc}$ in Korla pear based on the ANFIS model}

The method for predicting the Vc content in Korla pear based on the ANFIS model is consistent with that for SSC prediction. In the training stage, eight input MFs were chosen for fuzzification of the input data, and the linear MFs were selected for the output.
The error-tolerant rate was set as 0 and the iteration times was set as 100. The testing dataset was input into the trained model to obtain the prediction values. Figure 9 shows the correlations between the observed and predicted values in the training and prediction phases. The RMSE and $R^{2}$ values are listed in Table 4.

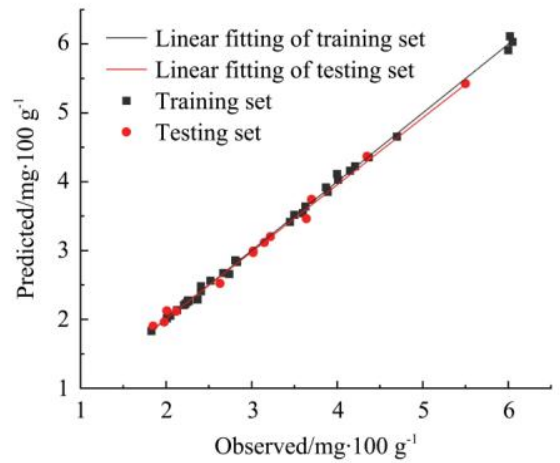

a. trimf

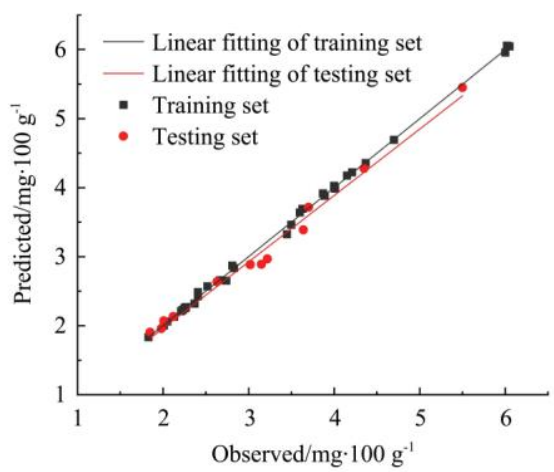

d. gaussmf

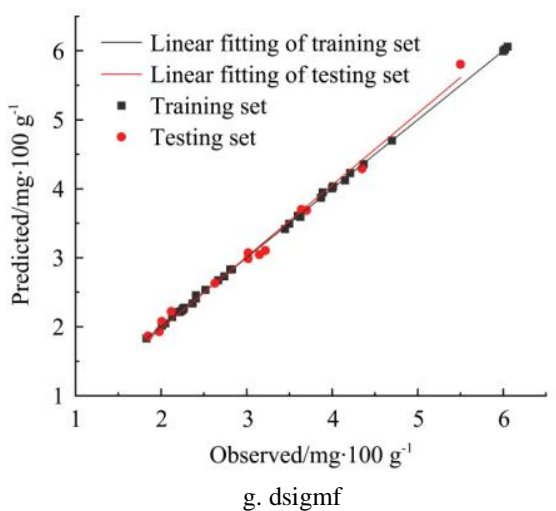

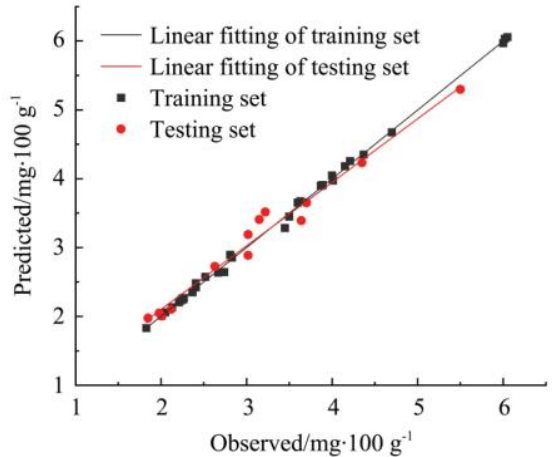

b. trapmf

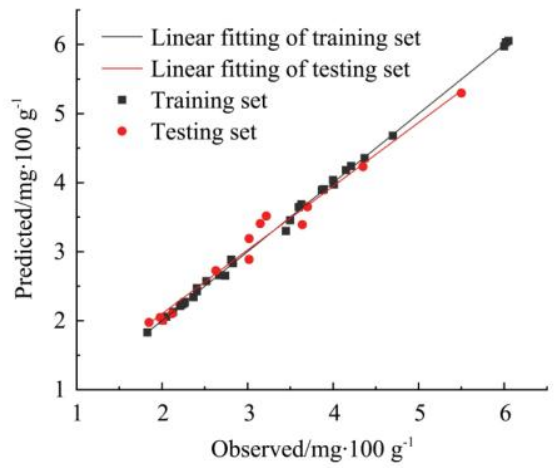

e. gasuss $2 \mathrm{mf}$

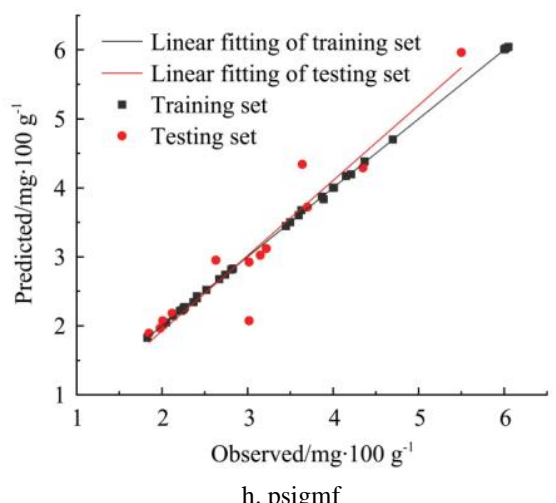

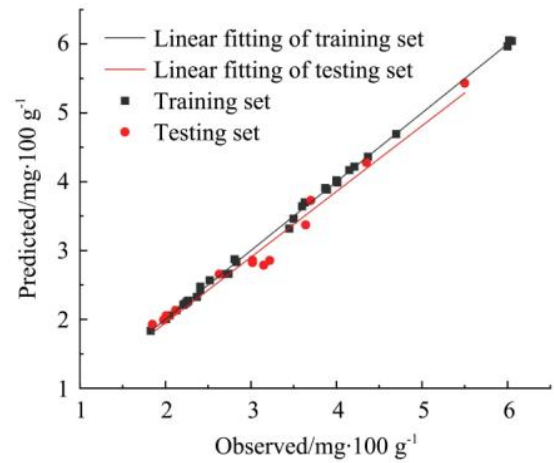

c. gbellmf

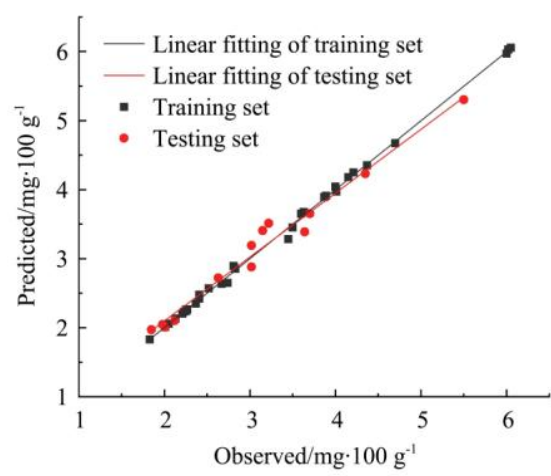

f. pimf

Figure 9 Correlations between the observed and predicted Vc content of Korla pear during storage by the ANFIS model with different MFs at the training and prediction phases

Table 4 Root mean squared error (RMSE) and coefficient of determination $\left(R^{2}\right)$ for Vc content prediction by the ANFIS model with different input MFs at the training and prediction phases

\begin{tabular}{|c|c|c|c|c|}
\hline \multirow{2}{*}{$\begin{array}{l}\text { Membership } \\
\text { function }\end{array}$} & \multicolumn{2}{|c|}{ Training stage } & \multicolumn{2}{|c|}{ Prediction stage } \\
\hline & RMSE & $R^{2}$ & RMSE & $R^{2}$ \\
\hline trimf & 0.045 & 0.99 & 0.075 & 0.99 \\
\hline trapmf & 0.047 & 0.99 & 0.164 & 0.97 \\
\hline gbellmf & 0.039 & 0.99 & 0.181 & 0.97 \\
\hline gaussmf & 0.041 & 0.99 & 0.138 & 0.98 \\
\hline gasuss $2 \mathrm{mf}$ & 0.043 & 0.99 & 0.164 & 0.97 \\
\hline pimf & 0.046 & 0.98 & 0.164 & 0.97 \\
\hline dsigmf & 0.020 & 0.99 & 0.105 & 0.99 \\
\hline psigmf & 0.016 & 0.98 & 0.368 & 0.91 \\
\hline
\end{tabular}

Table 4 shows that the $R^{2}$ values between the predicted values from the ANFIS model and the observed values are all higher than 0.91 , clearly demonstrating that the trained ANFIS model can effectively predict the changes of $\mathrm{Vc}$ content in Korla pear throughout the storage process under cold storage temperature. A comparative analysis showed that the ANFIS model which used trimf as the input MF has the best prediction performance.

3.3.4 Determination of the optimal prediction model

The two models obtained were used to predict the variation laws of SSC and Vc content in Korla pear in the storage process under cold storage temperature. It was found that when used to predict the SSC and Vc content in Korla pear, the GRNN model had a $R^{2}$ value of 0.97 ; and the ANFIS model had $R^{2}$ values higher than 0.91. Therefore, both the trained GRNN model and ANFIS model can well predict the SSC and Vc content in Korla pear in the 
storage process based on the data of harvest maturity and storage time. Moreover, the prediction performance of the GRNN model and ANFIS model was compared. The results suggest that the ANFIS model based on gbellmf is the most effective in predicting the SSC ( $\left.R M S E=0.175 ; R^{2}=0.98\right)$, while that based on trimf is the most effective in predicting the $\mathrm{Vc}$ content in Korla pear $\left(R M S E=0.075 ; R^{2}=0.99\right)$.

\subsubsection{Model verification}

A storage experiment under cold storage temperature was carried out in 2019 to verify the practical performance of the optimal prediction model. The SSC and Vc content in Korla pear fruits with the harvest maturity levels of $10 \%, 30 \%, 50 \%, 70 \%$ and $90 \%$ were determined after $30 \mathrm{~d}, 60 \mathrm{~d}$ and $120 \mathrm{~d}$ of storage. Linear fitting was then carried out between the observed and predicted values of the optimal models. The results are shown in Figure 10
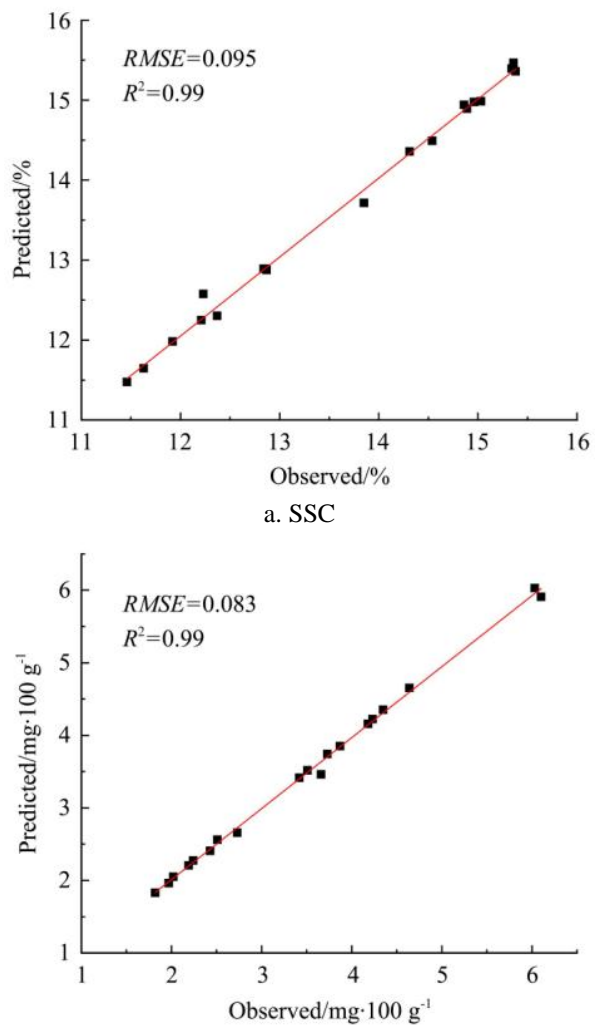

b. Vc content

Figure 10 Scatter plots of the observed values versus the predicted values for (a) SSC by the ANFIS model with gbellmf and

(b) Vc content by the ANFIS model with trimf

According to the verification experiment, the ANFIS model based on gbellmf can achieve a relatively higher prediction accuracy for SSC $\left(R M S E=0.095 ; R^{2}=0.99\right)$, while that based on trimf shows a relatively higher prediction accuracy for $\mathrm{Vc}$ content $\left(R M S E=0.083 ; R^{2}=0.99\right)$. These results suggest that the SSC and $\mathrm{Vc}$ content in Korla pear during storage can be predicted by imputing the data of harvest maturity and storage time into the optimal trained model.

\section{Conclusions}

It can be concluded that both harvest maturity and storage time can significantly influence the SSC and Vc content in Korla pear throughout the storage process under cold storage temperature. During cold storage, the SSC in pears with 10\%-70\% harvest maturity showed continuous increases in the first $90 \mathrm{~d}$ of storage and then a slight decline thereafter, while that in pears with $80 \%$ and $90 \%$ harvest maturity exhibited slow decreases throughout the storage process. With the extension of storage time, the $\mathrm{Vc}$ content of pears with $10 \%-90 \%$ harvest maturity showed continuous decreases.

The trained models can be used to effectively predict the changes of SSC and Vc content in Korla pear. The prediction performance of GRNN model was compared with that of ANFIS model under eight input MFs. It was found that the ANFIS model based on gbellmf had the highest prediction accuracy for SSC and that based on trimf can achieve the highest prediction accuracy for Vc content.

\section{Acknowledgements}

The authors express their acknowledgment to the Chinese Natural Science Foundation (Grant No. 31660475), Innovation and Entrepreneurship Project of the Xinjiang Production and Construction Group Special Commissioner for Science and Technology (Grant No. 2019CB037), University President Fund Project (Grant No. TDZKCQ201902), Xinjiang Production \& Construction Group Key Laboratory of Agricultural Products Processing in Xinjiang South (Grant No. AP1905), Young and Middle-aged Scientific and Technological Innovation Leaders Project of Xinjiang Production and Construction Group (Grant No. 2018CB014), Strong Youth Key Talents of Scientific and Technological Innovation (Grant No. 2021CB039) and Research Project of Double Employment Academician Work Funds Opening in Tarim University (Grant No. SPYS202002) for financial support and all of the persons who assisted in this writing.

\section{[References]}

[1] Wu J, Guo K Q. Dynamic viscoelastic behaviour and microstructura changes of Korla pear (Pyrus bretschneideri rehd) under varying turgor levels. Biosyst Eng, 2010; 106(4): 485-492.

[2] Wang B H, Sun X X, Dong F Y, Zhang F, Niu, J X. Cloning an expression analysis of an MYB gene associated with calyx persistence in Korla fragrant pear. Plant Cell Rep, 2014; 33(8): 1333-1341.

[3] Liu J, Zhang X, Li Z, Zhang X S, Jemric T, Wang X. Quality monitoring and analysis of Xinjiang 'Korla' fragrant pear in cold chain logistics and home storage with multi-sensor technology. Appl Sci, 2019; 9(18): 3895.

[4] Yu X J, Lu H D, Wu D. Development of deep learning method for predicting firmness and soluble solid content of postharvest Korla fragrant pear using Vis/NIR hyperspectral reflectance imaging. Postharvest Biol Technol, 2018; 141: 39- 49.

[5] Andrea C G S, Angel G, María I G. Comparative study of six pear cultivars in terms of their phenolic and vitamin $\mathrm{C}$ contents and antioxidant capacity. J Sci Food Agric, 2003; 83(10): 995-1003.

[6] Wang Z H, Wang W H, Jiang Y B, Bao A M, Tong W, Wang B X. Effects of different harvesting periods on the storage quality and senescence of apple at room temperature. Transactions of the CSAE, 2020; 36(7): 300-306. (in Chinese )

[7] Rizzolo A, Grassi M, Vanoli M. Influence of storage (time, temperature, atmosphere) on ripening, ethylene production and texture of 1-MCP treated 'Abbé Fétel' pears. Postharvest Biol Technol, 2015; 109: 20-29.

[8] Jia X H, Wang W H, Du Y M, Tong W, Wang Z H, Hera G. Optimal storage temperature and 1-MCP treatment combinations for different marketing times of Korla Xiang pears. J Integr Agric, 2018; 17(03): 693-703.

[9] Saquet A A. Storage of pears. Sci Hortic, 2019; 246: 1009-1016.

[10] Boonyakiat D, Chen P M, Spotts R A, Richardson D G. Effect of harvest maturity on decay and post-harvest life of 'd' Anjou' pear. Sci Hortic, 1987; 31: 131-139.

[11] Blanckenberg A, Muller M, Theron K I, Crouch E M, Steyn W J. Harvest maturity and ripeness differentially affects consumer preference of 'Forelle', 'Packham's Triumph' and 'Abate Fetel' pears (Pyrus communis L.). Sci Hortic, 2016; 207: 131-139.

[12] Dong Y, Zhang S Y, Wang Y. Compositional changes in cell wall 
polyuronides and enzyme activities associated with melting/mealy textural property during ripening following long-term storage of 'Comice' and 'd'Anjou' pears. Postharvest Biol Technol, 2018; 135: 131-140.

[13] Nordey T, Davrieux F, Léchaudel M. Predictions of fruit shelf life and quality after ripening: Are quality traits measured at harvest reliable indicators? Postharvest Biol Technol, 2019; 153: 52-60.

[14] Huang Y B, Lan Y B, Thomson S J, Fang A, Hoffmann W C, Lacey R E. Development of soft computing and applications in agricultural and biological engineering. Comput Electron Agr, 2010; 71: 107-127.

[15] Guo W C, Shang L, Zhu X H, Shang L, Nelson S O. Nondestructive detection of soluble solids content of apples from dielectric spectra with ANN and chemometric methods. Food Bioprocess Tech, 2015; 8(5): $1126-1138$.

[16] Jiang Z D, Zheng H, Mantri N, Qi Z C, Zhang X D, Hou Z N, et, al. Prediction of relationship between surface area, temperature, storage time and ascorbic acid retention of fresh-cut pineapple using adaptive neuro-fuzzy inference system (ANFIS). Postharvest Biol Technol, 2016; 113: $1-7$

[17] Zheng H, Jiang B, Lu H F. An adaptive neural-fuzzy inference system (ANFIS) for detection of bruises on Chinese bayberry (Myrica rubra) based on fractal dimension and RGB intensity color. J Food Eng, 2011; 104: 663-667

[18] National Standard of the People's Republic of China. Korla fragrant pear, 2002; NY/T 585-2002. (in Chinese)

[19] National Standard of the People's Republic of China. Production technical rules for Korla fragrant pear, 2004; NY/T 881-2004. (in Chinese)

[20] Lan H P, Jia F G, Tang Y R, Zhang Q, Han Y L, Liu Y. Quantity evaluation method of maturity for Korla fragrant pear. Transactions of the CSAE, 2015; 31(5): 325-330. (in Chinese)

[21] Guo W C, Fang L J, Liu D Y, Wang Z W. Determination of soluble solids content and firmness of pears during ripening by using dielectric spectroscopy. Comput Electron Agr, 2015; 117: 226-233.

[22] Nicolaï B M, Verlinden B E, Desmet M, Saevels S, Saeys W, Theron K, et al. Time-resolved and continuous wave NIR reflectance spectroscopy to predict soluble solids content and firmness of pear. Postharvest Biol Technol, 2008; 47(1): 68-74.
[23] Peiris K H S, Dull G G, Leffler R G, Kays S J. Spatial variability of soluble solids or dry-matter content within individual fruits, bulbs, or tubers: implications for the development and use of NIR spectrometric techniques. HortScience, 1999; 34(1): 114-118.

[24] Paz P, Sánchez M T, Pérez-Marín D, Guerrero J E, Garrido-Varo A Instantaneous quantitative and qualitative assessment of pear quality using near infrared spectroscopy. Comput Electron Agr, 2009; 69(1): 24-32.

[25] Wang Z H, Wang W H, Jiang Y B, Bao A M, Tong W, Wang B X Effects of different harvesting periods on the storage quality and senescence of apple at room temperature. Transactions of the CSAE, 2020; 36(7): 300-306. (in Chinese )

[26] Tijskens L M M, Konopacki P, Simcic M. Biological variance, burden or benefit? Postharvest Biol Technol, 2003; 27(1): 15-25.

[27] Heddam S. Generalized regression neural network based approach as a new tool for predicting Total Dissolved Gas (TDG) downstream of spillways of dams: a case study of Columbia river basin dams, USA. Environ Process, 2017; 4: 235- 253.

[28] Li H. Statistic learning method (second edition). Beijing: Tsinghua University Press, 2019; 24p. (in Chinese)

[29] Rooki R. Application of general regression neural network (GRNN) for indirect measuring pressure loss of Herschel-Bulkley drilling fluids in oil drilling. Measurement, 2017; 85: 184-191.

[30] Jang J S. ANFIS: Adaptive-network-based fuzzy inference system. IEEE T Syst Man Cy-s, 1993; 23(3): 665-685.

[31] Liu X. Studies on mechanism of fruit growth and development of different ripening-season of pears in China. $\mathrm{PhD}$ dissertation. Ya'an: Sichuan Agricultural University, 2008; 6. 48p. (in Chinese)

[32] Shao M C. Studies on technique for 'Hosui' pear storage in China. Master dissertation. Nanjing: Nanjing Agricultural University, 2005; 12. 16 p. (in Chinese)

[33] Li G L. Study on the preservation technique of Huanghua pear in China. Master dissertation. Fuzhou: Fujian Agricultural and Forestry University, 2013; 4. 27p. (in Chinese)

[34] Lee S K, Kader A A. Preharvest and postharvest factors influencing Vitamin C content of horticultural crops. Postharvest Biol Technol, 2000; 20(3): 207-220. 